\title{
Familiarity and visual change detection
}

\author{
HAROLD PASHLER \\ University of California, San Diego, La Jolla, California
}

\begin{abstract}
Detection of change when one display of familiar objects replaces another display might be based purely upon visual codes, or also on identity information (i.e., knowing what was present where in the initial display). Displays of 10 alphanumeric characters were presented and, after a brief offset, were presented again in the same position, with or without a change in a single character. Subjects' accuracy in change detection did not suggest preservation of any more information than is usually available in whole report, except with the briefest of offsets (under 50 msec). Stimulus duration had only modest effects. The interaction of masking with offset duration followed the pattern previously observed with unfamiliar visual stimuli (Phillips, 1974). Accuracy was not reduced by reflection of the characters about a horizontal axis, suggesting that categorical information contributed negligibly. Detection of change appears to depend upon capacity-limited visual memory; (putative) knowledge of what identities are present in different display locations does not seem to contribute.
\end{abstract}

The goal of the present study was to characterize observers' abilities to detect changes in visual displays of items that have disappeared only briefly. Phillips (1974) investigated change detection in displays of unfamiliar visual stimuli, and provided strong evidence that performance is mediated by two systems: sensory persistence and visual short-term memory. An obvious possibility of interest is that when highly familiar items are presented, information about the identities of the characters can also contribute to the detection of change. It is widely supposed that the identities and the locations of familiar objects in the visual field are encoded rapidly and automatically (e.g., Duncan, 1980; Shiffrin, 1976), and that a comparable level of encoding is not available for unfamiliar stimuli (e.g., LaBerge \& Samuels, 1974). Thus, one very natural possibility is that identities might make a powerful contribution to performance in the changedetection task; that is, the subject might detect change not only by comparing visual representations derived from the sequence of displays, but also by detecting changes in what object identities are present in particular locations. In view of the wide range of studies that have investigated recognition memory, successive matching, and visual sensory persistence, it is surprising that this possibility does not seem to have been investigated in any systematic way. In the experiments reported here, accuracy of change detection was studied as a function of a number of variables, including stimulus duration, interstimulus interval (ISI), intervening masks, and, most crucially, the composition of the display (familiar vs. unfamiliar characters).

The author is grateful to James C. Johnston and Jeff Miller for useful discussions, and to Jeff Hartung and Chris Casella for assistance in conducting the experiments reported here. Lester Krueger, Robert Proctor, and Richard Shiffrin provided very useful comments on an earlier version of the manuscript. Correspondence should be addressed to Harold Pashler, Department of Psychology, C-009, University of California, San Diego, La Jolla, CA 92093.
As noted above, the literature contains a large amount of work on various related tasks. Matching of letter strings has been extensively studied, and some comprehensive theories of matching have been proposed (e.g., Krueger, 1978, 1984; Proctor, 1981). Some of this research concerns matching of successively presented strings. This work, however, has been explicitly concerned mostly with cognitive matching processes in situations designed to preclude possible involvement of sensory persistence; thus, the second display has typically been presented in a location distant from that of the first display (a notable exception is a study by Purdy, Eimann, \& Cross, 1980 , described below). Matching work, therefore, has not systematically explored the ability of subjects to detect changes in the composition of alphanumeric displays over short intervals of time, when the second display occurs in the same spatial and retinal location as the first. Given these task differences, the present paradigm is termed change detection, rather than matching (or recognition memory), despite the fact that, logically speaking, the subject's task is the same.

Change detection with unfamiliar visual stimuli was thoroughly studied by Phillips (1974), who carried out an elegant series of experiments aimed at exploring differences between sensory memory and visual short-term memory. Phillips's experiments required subjects to detect changes in displays consisting of partially filled grids of dots. For example, a typical display consisted of 12 dots occupying positions in a $5 \times 5$ matrix. The display that followed either was identical to the first or differed in the addition or removal of a single dot. The data showed that excellent peformance, even with large displays, was accomplished when the ISI between the dots was short (less than about $100 \mathrm{msec}$ ). This performance was grossly disrupted by interposing a mask between the diplays or by translating the test display in the visual field. With longer ISIs, performance was not nearly as good, but it 
was not affected very substantially by masking, and was affected hardly at all by translation. Phillips suggested that sensory memory was responsible for the excellent short ISI performance; this system, he suggested, was clearly maskable (a result that is consistent with a variety of other evidence; e.g., Averbach \& Coriell, 1961). The performance at longer ISIs was attributed to visual short-term storage, relatively unmaskable and apparently usable despite display translation. In subsequent studies, Phillips and his colleagues further investigated the characteristics of this system (Avon \& Phillips, 1980; Phillips \& Christie, 1977).

Phillips's (1974) work provided a convincing dissection of two distinct contributions to change detection, using stimuli that are basically unfamiliar and that lack names. Given the various theories that have been advanced concerning the possible role of abstract representations in tasks involving familiar stimuli such as alphanumeric characters, it is natural to wonder whether the performance in change detection with familiar stimuli would show patterns similar to those observed with Phillips's dot matrices, or whether categorical information would alter the performance. If subjects do, in fact, automatically analyze visual stimuli and create a representation that contains identity and location information, appropriately tied together, then these postcategorical representations may well produce a dramatic enhancement of change detection; that is, the subject may be able to tell that a change occurred by detecting a difference in the mapping of abstract identities to locations in the display.

Excellent performance in change detection with familiar characters has, in fact, been claimed. Purdy et al. (1980) reported an investigation of change detection in letter displays. They presented two 100 -msec displays of 16 letters, separated by ISIs ranging from $100 \mathrm{msec}$ to $5 \mathrm{sec}$. The second display contained no changes, or changed in 1,4 , or 16 positions. The authors found very little decrement in performance as the ISI was lengthened, and change detection was very good $(95 \%)$ when all 16 letters were changed. They interpreted their data as evidence for long-lasting sensory memory, and stated that it is " interesting that evidence for an extended sensory memory only occurs when using the same-different recognition, [since it] was not shown in Sperling's recall paradigm" (p. 376).

Purdy et al. (1980) apparently attributed performance in their task to a specifically sensory memory, and did not attribute it to the presence of categorical information per se. However, their interpretations can be questioned in two ways. A close examination of their data suggests that the level of performance observed may not be as impressive as they suggested. When all 16 positions were changed, accuracy of detection was indeed nearly perfect; however, perfect performance would be obtained in this condition if subjects preserved only a single character and monitored its position for a change. A better indication of the performance level would seem to be provided by change-detection accuracy when a single character was changed from the first to the second display. In this condition, at the 100 -msec ISI, the subjects in Purdy et al.'s study had a hit rate of .42 and a false-alarm rate of .15. One way of quantifying this performance is as follows: Suppose the subjects performed the task by "holding on to" a particular number of characters $(k)$; when one of these characters changed, a correct detection would occur. When no change was thus detected, the subject would say "no," except on some proportion of trials (g) on which he/she would guess "yes." In this case, the hit rate (H) would be

$$
H=k / 16+\left[(16-k) / 16^{*} g\right],
$$

and the false alarm rate $(F A)$ would be simply

$$
F A=g
$$

This simple model is admittedly crude in admitting no partial information and in attributing all errors to the maintenance, rather than the comparison, process. However, it seems to provide one reasonable description of the level of performance, correcting for guessing in a natural way. When this simple model is fit to the Purdy et al. (1980) data just listed, it indicates that subjects were holding on to about five digits (i.e., $k=5.08$ ). This is very close to the level typically observed in whole-report data (Sperling, 1960). As such, the model suggests a very different perspective on the results from that advanced by Purdy et al: when subjects merely have to detect a change in a display over a brief offset, their accuracy indicates preservation of not much more than the subjects might actually have produced in whole report. This is quite surprising, especially since whole report would seem to be a more demanding task in several respects. Since superiority to the level predicted by whole-report performance is the conventional index of sensory memory involvement, the data reported by Purdy et al. do not seem to require their invocation of "extended sensory memory." Storage in a capacity-limited short-term memory system would seem quite compatible with the overall level of performance achieved by their subjects.

It would not be appropriate, however, to conclude from the results of Purdy et al. (1980) that subjects cannot perform substantially better than this in change detection with familiar items. One factor that could have made Purdy et al.'s task particularly difficult is the very short (100$\mathrm{msec}$ ) exposure durations they employed. Even if abstract identity information (i.e., knowledge of what was present where) is capable of producing enhanced change detection, such information might well take several hundred milliseconds to extract. It is difficult to determine exactly how long such processing takes, because one cannot be sure of instantaneously terminating it with masks or other known manipulations.

We began, therefore, by examining performance as a function of display duration, to determine whether the Purdy et al. (1980) result is reliable and whether it depends strongly on their use of short displays. We then 
asked how performance changes with ISI, to determine whether superior performance can be observed at short ISIs, as Phillips (1974) found with his stimuli. In addition, the effects of masks were examined over the ISI range. The final two experiments attempted to test the conjecture that performance in this task might depend purely upon visual codes, by asking whether minimizing the role of identity information by mirror reflecting the letters would impair performance.

\section{EXPERIMENT 1}

Experiment 1 examined performance in the detection of changes in character displays as a function of exposure duration. Displays consisted of 10 consonants presented in two rows of 5 characters; adjacent items were separated by one character space both horizontally and vertically. Displays were refreshed at $60 \mathrm{~Hz}$ for 100,300 , or $500 \mathrm{msec}$, and, after a $67-\mathrm{msec}$ offset, were presented again for the same duration in the same position; half the time, one letter was changed in the second display.

\section{Method}

Subjects. Fifteen undergraduates at the University of California, San Diego, participated as subjects in the experiment, in partial fulfillment of a course requirement.

Apparatus and Stimuli. The stimuli were presented on an Amdek Color-I CRT, and a Commodore microcomputer controlled the stimulus presentations and collected the responses. The computer was programmed in machine language so as to obtain synchrony with the $60-\mathrm{Hz}$ refresh scan of the CRT. Each display consisted of 10 letters, presented in two rows of 5 white letters against a black background. The brightness of the letters and background were approximately $141 \mathrm{~cd} / \mathrm{m}^{2}$ and $1 \mathrm{~cd} / \mathrm{m}^{2}$, respectively. ${ }^{1}$ Each character was $7 \mathrm{~mm}$ high and $4 \mathrm{~mm}$ wide. Based on a typical viewing distance of $70 \mathrm{~cm}$, this represented $.6^{\circ} \times .3^{\circ}$ visual angle. The entire display was $4.9 \mathrm{~cm}$ tall and $2.3 \mathrm{~cm}$ wide $\left(4.0^{\circ} \times 1.9^{\circ}\right)$, and the spacing between each character was approximately $.7 \mathrm{~cm}$ horizontally $\left(.6^{\circ}\right)$ and $.8 \mathrm{~cm}$ vertically $\left(.6^{\circ}\right)$. The displays were composed by selecting independently and with replacement from the 20 consonants. The exclusion of vowels (including $Y$ ) was to reduce the probability of any of the displays spelling out words, which has marked effects in related tasks (Lefton, 1973). The second display either was completely identical to the first or differed from it in the identity of one character. When it differed, the character was replaced with a different character chosen at random from the stimulus set, without regard to whether that character appeared elsewhere in the display.

Design. The experiment was divided into nine blocks, each of which consisted of 20 trials with no change and 20 trials with a change. For the latter trials, the change occurred equally often (twice) in each of the 10 possible positions. The exposure duration of the first and second displays $(100,300$, or 500 msec each) was varied between blocks, and the exposure duration rotated from block to block, in the order $100,300,500,100,300$, and so forth. The subjects were divided into three conditions, based on the exposure duration of their first (and thus subsequent) blocks. Thus, duration was not confounded with average position in the experiment. The presentation order was individually randomized at the beginning of each block.

Procedure. The subjects were given written instructions to inform them that their task was to detect any changes in items composing a display, over a brief offset. The importance of accuracy and the unimportance of speed were emphasized. Before the ex- periment itself, the subjects completed one block of practice trials, sampling the various conditions.

The experiment was conducted with ordinary room lighting. The experiment began with the presentation of a fixation point (a plus sign) in the center of the screen. This remained present for $500 \mathrm{msec}$. At its offset, the fixation point was replaced with the display of 10 characters. The display was refreshed for the preset exposure duration $(100,300$, or $500 \mathrm{msec}$ for the whole block). After a $67-$ msec interval, the display reappeared for the same duration and in the same position in which it initially appeared, either with or without a single change in one position. (The $67-\mathrm{msec}$ figure represents the interval between the last refresh of the first display and the first refresh of the second display; i.e., there were three successive blank refreshes of the $60-\mathrm{Hz}$ cycle.) The subject responded by pressing one of two keys on the keyboard, the $\mathbf{N}$ key for "same" or the M key for "different" (both keys were clearly labeled), using the index or middle finger of the right hand, respectively. The words "CORRECT" or "WRONG" appeared on the screen (slightly below the position of the letter displays), 300 msec after the subject's response and remained present for $500 \mathrm{msec}$. The overall intertrial interval, measured from the subject's response to the appearance of the fixation point for the next trial, was $2.95 \mathrm{sec}$.

\section{Results}

Table 1 presents subjects' average performance in detecting changes as a function of display duration and position of change. The overall percentages of correct responses were $59.6 \%, 65.4 \%$, and $69.0 \%$ for displays of 100,300 , and $500 \mathrm{msec}$, respectively. In addition, a sensitivity $\left(d^{\prime}\right)$ analysis of each subject's accuracy on the three durations (pooled across position of change) was performed. The mean $d^{\prime}$ s were $0.588,0.848$, and 1.059 for durations of 100,300 , and $500 \mathrm{msec}$, respectively. These $d^{\prime}$ results were subjected to an analysis of variance (ANOVA), and the effect of stimulus exposure duration was significant $[F(2,28)=9.76, p<.001]$.

\section{Discussion}

The results of Experiment 1 indicate that subjects' performance in detecting single changes in character displays is remarkably poor when a 67 -msec offset separates the first and second display. There is a reliable but modest improvement in performance as exposure duration is increased from 100 to $500 \mathrm{msec}$. Thus, the poor perfor-

Table 1

Proportion of Correct Responses as a Function of Stimulus Duration in Experiment 1

\begin{tabular}{cccc}
\hline & \multicolumn{3}{c}{ Stimulus Duration } \\
\cline { 2 - 4 } Position of Change & $100 \mathrm{msec}$ & $300 \mathrm{msec}$ & $500 \mathrm{msec}$ \\
\hline None & .66 & .73 & .74 \\
1 & .61 & .66 & .63 \\
2 & .49 & .62 & .67 \\
3 & .71 & .78 & .78 \\
4 & .56 & .60 & .69 \\
5 & .61 & .53 & .56 \\
6 & .47 & .52 & .58 \\
7 & .31 & .46 & .54 \\
8 & .57 & .62 & .70 \\
9 & .46 & .54 & .61 \\
10 & .53 & .48 & .60 \\
$1-10$ & .53 & .58 & .64 \\
\hline
\end{tabular}


mance observed by Purdy et al. (1980) with the same-different task is not due only to their use of brief displays. In addition, decreasing the ISI from $100 \mathrm{msec}$ to the $67 \mathrm{msec}$ employed here does not appear to have improved performance.

As noted above, Purdy et al. (1980) regarded performance in their experiments as indicative of high-capacity sensory preservation, that is, the storage thought to underlie the partial-report superiority effect. Apparently, this conclusion was based on the accuracy achieved when the second display differed from the first in all positions. However, as noted, an analysis of the one-item-change condition in Purdy et al.'s work suggests a contrary conclusion: the accuracy levels are consistent with preservation of no more information than is characteristically available in whole report. The same is true of the present data, all of which concern single-item changes. Consider, for instance, the $300-\mathrm{msec}$ duration condition in the present experiment: subjects detected $58.1 \%$ of all changes, and incorrectly reported a change on $27.2 \%$ of the trials in which no change occurred. The simple model described earlier can be applied here as well. If, as mentioned above, one assumes that subjects hold on to a certain number of items, always detecting a change when it occurs in one of those positions and guessing that a change has occurred a certain proportion of the time when no change is detected, then the data estimate that subjects maintained 4.24 items. As mentioned earlier, the discreteness implied by this model is, no doubt, not strictly correct; nonetheless, the model seems to provide a reasonable metric by which to compare performance in the present task with wholereport performance. The clear conclusion from such a comparison is that change detection reveals surprisingly limited preservation of information.

\section{EXPERIMENT 2}

Experiment 1 showed that accuracy in detecting change in character displays over very brief offsets shows that little more information is preserved than is typically available in whole report; the result was not much affected by display duration. The present experiment examines the effect of ISI and masking, jointly manipulated. As described in the introduction, Phillips (1974) observed a strong interaction between masking and ISI in a task that required subjects to judge whether two displays of dots were the same or different. Specifically, at very short ISIs, performance was excellent unless a mask was present. At longer ISIs (100 msec and longer), performance leveled off, and masking effects were notably weaker. Phillips suggested that sensory memory, especially vulnerable to masking, was responsible for the excellent performance at short ISIs; at longer ISIs, such traces decayed to the point of being unusable. At the longer ISIs, Phillips suggested, visual short-term storage must be relied upon. If performance in the present task depends upon the same mechanisms as those underlying change detection with Phillips's dot patterns, we might expect very similar results when masking is introduced in the paradigm ex- plored in the present work. Alternatively, the pattern might be quite different due to the use of categorizable familiar stimuli in the present work.

The experiment required subjects to detect single changes in 300-msec letter strings, exposed in the same positions and separated by delays of 34,67 , and $217 \mathrm{msec}$. During this time, either mask characters (white homogeneous or checkerboard squares) or the dark background field were interposed in the same positions as the white characters.

\section{Method}

Subjects. Twenty-four undergraduates at the University of California, San Diego, participated as subjects in the $1-h$ experiment, in partial fulfillment of a course requirement.

Apparatus and Stimuli. The apparatus and stimuli were nearly identical to those of Experiment 1. The mask characters were squares of approximately the same outer dimensions as the characters. In the mask condition, 10 mask characters appeared for the duration of the offset interval, in the positions occupied by the display characters. Two different types of mask characters were used. In one mask condition (checkerboard mask), a $4 \times 4$ black-and-white checkerboard pattern was presented in each position. In the other mask condition (homogeneous mask), a pure white square was presented in each position.

Design and Procedure. The experiment was divided into 12 blocks, each of which consisted of 20 trials with no change and 20 trials with a change. For the latter trials, the change occurred equally often (twice) in each of the 10 possible positions. Throughout the experiment, the exposure duration of the first and second displays was set at $300 \mathrm{msec}$. Half of the subjects were assigned to the grid mask condition, and half to the white mask condition. There were six types of blocks, representing the three ISIs $(34,67$, and $217 \mathrm{msec}$ ) crossed with mask (present or absent). The 12 blocks in the experiment cycled through the six block types. Within each of the two mask-type groups, subjects were assigned to one of six conditions, determining initial block type. The presentation order was individually randomized at the beginning of each block. The general procedure followed that of Experiment 1, except for the presence of the 10 mask characters during the offset in the mask condition.

\section{Results}

Table 2 presents subjects' average performance in detecting changes as a function of masking and ISI. In addition, a sensitivity $\left(d^{\prime}\right)$ analysis of each subject's accuracy on the translation conditions (pooled across position of change) was performed. These average sensitivity scores are presented in Figures 1 and 2, representing perfor-

Table 2

Proportion of Correct Responses as a Function of Interstimulus Interval and Masking in Experiment 2

\begin{tabular}{|c|c|c|c|c|c|c|}
\hline \multirow[b]{3}{*}{ Change } & \multicolumn{6}{|c|}{ Interstimulus Interval } \\
\hline & \multicolumn{2}{|c|}{$34 \mathrm{msec}$} & \multicolumn{2}{|c|}{$67 \mathrm{msec}$} & \multicolumn{2}{|c|}{$217 \mathrm{msec}$} \\
\hline & No Mask & Mask & No Mask & Mask & No Mask & Mask \\
\hline \multicolumn{7}{|c|}{ Homogeneous Mask } \\
\hline No & .91 & .75 & .75 & .68 & .70 & .61 \\
\hline Yes & .81 & .51 & .61 & .50 & .58 & .52 \\
\hline \multicolumn{7}{|c|}{ Checkerboard Mask } \\
\hline No & .87 & .79 & .68 & .82 & .72 & .73 \\
\hline Yes & .79 & .44 & .63 & .33 & .55 & .43 \\
\hline
\end{tabular}


mance with the homogeneous and checkerboard masks, respectively. An ANOVA was performed on the sensitivity scores. The effect of ISI was significant $[F(2,44)$ $=56.3, p<.001]$, as was the effect of presence of a mask $[F(1,22)=60.6, p<.001]$. The effects of ISI and mask presence interacted $[F(2,44)=33.4, p<.001]$. The effect of mask type (checkerboard vs. homogeneous), however, was nonsignificant $[F(1,22)=0.6, p>.40]$. Furthermore, there were no indications of any interactions involving mask type.

\section{Discussion}

The results show a clear interaction: performance was excellent at the shortest ISI ( $34 \mathrm{msec}$ ), but only when no mask was presented. When a mask was presented at the short ISIs, performance was brought down near the level of the longer ISI conditions. Performance did not deteriorate much from $67 \mathrm{msec}$ to $217 \mathrm{msec}$, and there was a clear, but much more modest, effect of masking in these conditions. There is no evidence that the presence of contours in the mask had much effect on the results (although Table 2 suggests some tendency for the checkerboard mask to bias subjects against reporting change).

This pattern of effects is very close to what Phillips (1974) observed with nonverbalizable dot patterns, although the pattern unfolds over a slightly different time course than in his observations. Phillips found evidence of sensory storage (excellent, but maskable, performance) at ISIs up to $100 \mathrm{msec}$ in his task, whereas in the present experiments such rapidly decaying storage is apparently unusable by the 67-msec ISI, since performance at the 67-msec ISI appears virtually the same as that at the 217msec ISI.

It appears, then, that at short ISIs (here, $34 \mathrm{msec}$ ), some form of highly maskable sensory storage permits excellent performance in the present task. Performance stabilizes at $67 \mathrm{msec}$ at the relatively poor level observed in

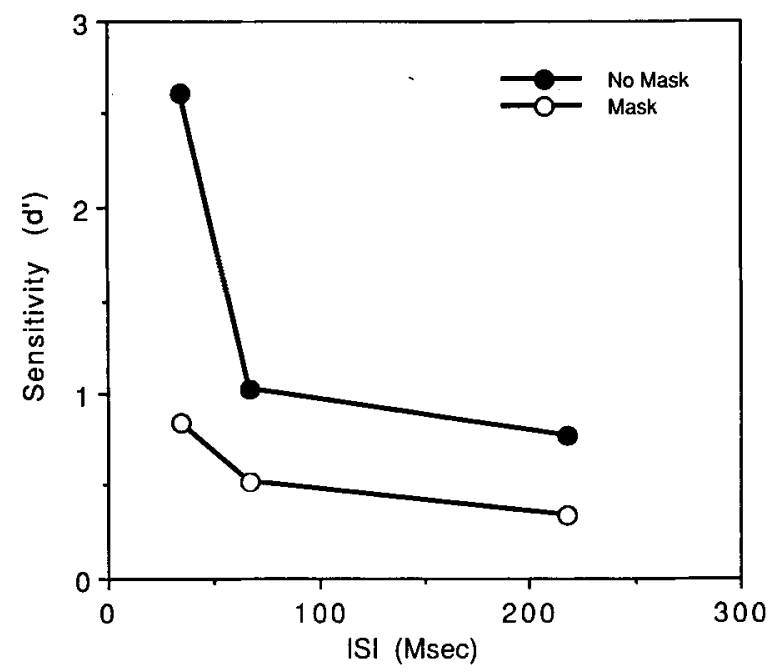

Figure 1. Sensitivity as a function of interstimulus interval (ISI) and masking for the homogeneous mask condition in Experiment 2.

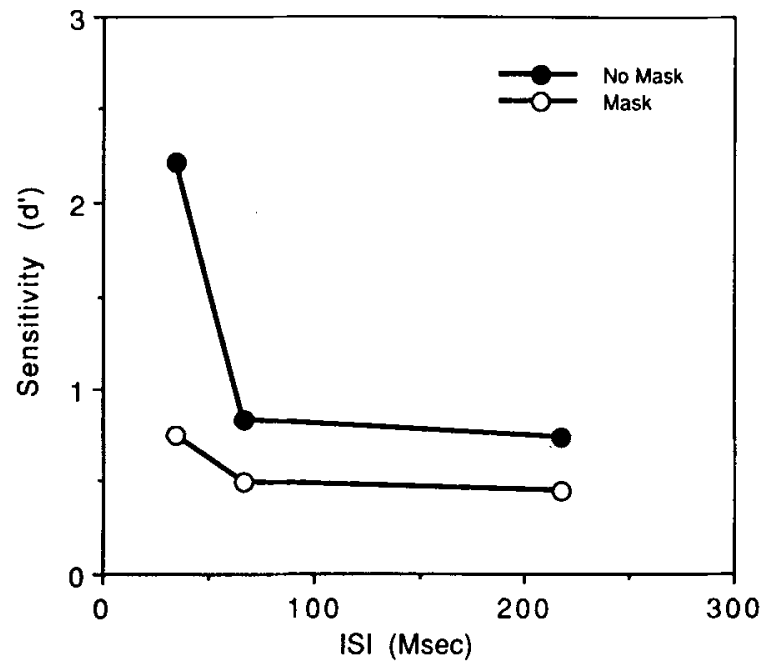

Figure 2. Sensitivity as a function of interstimulus interval (ISI) and masking for the checkerboard mask condition in Experiment 2.

the previous experiment, and mask effects are smaller, although not negligible, here. Mask effects were still apparent at long ISIs in Phillips's (1974) data as well. In particular, Phillips's Experiment 3 jointly manipulated masking and ISI in change detection with dot patterns drawn in $5 \times 5$ or $8 \times 8$ elements. The mask effects were most dramatic at the shortest ISIs, as in the present data. Mask effects seemed to virtually disappear at long ISIs for the $8 \times 8$ displays, but not for the $5 \times 5$ displays; thus, the present data look more like Phillips's $5 \times 5$ dot patterns. It is not clear why the mask effects seemed to disappear with Phillips's $8 \times 8$ displays, and he does not offer any account of this aspect of his data. In summary, then, the present data are very similar to those of Phillips, whose work examined change detection with dot patterns; the only exception to this similarity is Phillips's unexplained finding that masking effects appeared to be abolished at long ISIs, with the more, but not with the less, complex of his dot patterns. In the present experiment, masking effects were reduced with the longer ISIs, but never seem to have disappeared.

The overall data suggest that performance at the very short ISIs may be based upon some form of relatively peripheral sensory information, that is, in Phillips's (1974) terms, sensory storage. This raises the interesting question of how such sensory storage might subserve change detection. The most obvious suggestion, considered by Phillips, is that the short ISI changes trigger perception of apparent motion. Phillips rejected the motion hypothesis on the basis of his finding that the high accuracy performance with very short ISIs could not be obtained with dichoptic presentations; apparent motion, however, is known to occur dichoptically. Phillips suggested instead that interactions between more peripheral offset and onset detectors might underlie the high accuracy with short ISIs. These hypotheses will not be considered further here, but let me point out that motion might possibly play a role 
in the present task with short ISIs, even if it did not contribute in Phillips's task, because of the nature of the stimulus configurations. When a display changed in Phillips's task, a dot either appeared or disappeared; neither of these may provide an optimal stimulus for motion detection. On the other hand, in the present work, when a character changed, motion of very local (i.e., withincharacter) contours may have been perceived. The asymptotic performance (at 67- and 217-msec ISIs) is presumably due to something other than sensory memory. Visual short-term memory (as delineated by Phillips) is a natural candidate.

The fact that there are still substantial mask effects at these longer ISIs does not gainsay this account, since masks might well disrupt a change-detection task in ways other than destroying sensory memory. For instance, the comparison process might not completely succeed in avoiding treating the mask as a target stimulus to some extent. This could generate a kind of Stroop-like effect. Furthermore, masks might impair performance by disrupting sensory memory even if sensory memory itself was not useful in the change detection at a given ISI, because the masks would shorten the effective duration of the first stimulus. Experiment 1 indicated that performance continues to improve gradually as duration is lengthed to $1 / 2 \mathrm{sec}$. This suggests that a blank ISI would be useful simply in allowing encoding (perhaps into visual short-term memory) to proceed during the ISI.

\section{EXPERIMENT 3}

Experiment 1 demonstrated that performance at detecting changes in chàracter displays over very brief offsets was not much better than would be expected on the basis of typical whole-report performance. Experiment 2, which jointly manipulated ISI and presence of an intervening mask, found results very much like those observed by Phillips (1974) using non-nameable dot patterns. Together, these results encourage the suspicion that performance in tasks that require subjects to detect changes in character displays may depend only upon the two brief visual memory systems that Phillips delineated. That is, any abstract information about which characters are present in the display may, for some reason, play no role in the change-detection paradigm. These results plainly do not demonstrate that identities are playing no role, however. A more direct test is required.

If the hypothesis that identity information plays no role in the task should be correct, we might expect that the task could be performed as well with unfamiliar, nonidentifiable forms as with highly familiar forms such as alphanumeric characters. One test of the hypothesis, then, would be to create an "artificial" character set, and determine whether subjects perform as well with these characters as with the usual alphanumeric set. Unfortunately, such an experiment is problematic, since there is no obvious way to be sure that the average visual complexity and similarity of pairs of items within an artificial character set approximates that found within the standard alphanumeric character set. In Experiment 3, we attempted to surmount the problem of controlling the visual changes that comprise the display changes by using distorted alphanumeric characters. A similar approach was used in a study by Reicher, Snyder, and Richards (1976) in examining the effects of familiarity in visual search, and in other studies reviewed by Krueger (1975). Specifically, we compared subjects' accuracy in detecting changes in arrays of characters in their familiar rightside-up orientation with their accuracy in detecting changes in arrays of characters that were reflected about their horizontal axis. Only characters that are asymmetric about their horizontal axis were utilized in this experiment. (It should be noted that this transformation is, of course, not equivalent to a $180^{\circ}$ rotation, except in the case of characters that happen to be symmetric about a vertical axis.) Reflection appears to reduce the identifiability of the characters fairly grossly, and thus if performance in the change-detection task depends to any appreciable degree upon character identities, a substantial decrement in performance would be expected. However, reflection seems likely to leave the visual confusability of pairs within each character set largely unchanged.

The present experiment, then, compared changedetection performance in normal character and reflectedcharacter displays; the two displays each lasted $300 \mathrm{msec}$, and were separated from each other by an ISI of 34,67 , or $217 \mathrm{msec}$.

\section{Method}

Subjects. Eighteen undergraduates at the University of California, San Diego, participated as subjects in the 1-h experiment, in partial fulfillment of a course requirement.

Apparatus and Stimuli. The apparatus and stimuli were nearly identical to those of Experiment 1. The main difference was in the stimuli used in the reflected-character blocks. Reflection was accomplished by altering the character set specifications in the microcomputer. The characters are specified by an $8 \times 8$ pixel array; for the reflected characters, a switch was performed of the 1 st and 8 th rows or pixels, the 2 nd and 7 th rows, and so on. The following uppercase characters were employed in the experiment: $F, G, J$, $\mathrm{L}, \mathrm{N}, \mathrm{Q}, \mathrm{R}, \mathrm{T}, \mathrm{V}, \mathrm{Y}, \mathrm{Z}$, and 2 . They were selected to ensure that reversal did not leave the character unchanged and did not produce a different, but recognizable character.

Design and Procedure. The experiment was divided into 18 blocks, each of which consisted of 10 trials with no change and 10 trials with a change. For the latter condition, the change $o c-$ curred equally often (once) in each of the 10 possible positions. Throughout the experiment, the exposure duration was set at $300 \mathrm{msec}$. There were six types of blocks, representing the three ISIs $(34,67$, and $217 \mathrm{msec})$ crossed with the two types of display (normal and reflected). The 18 blocks in the experiment cycled three times through the six block types. Each subject was assigned to one of six conditions, determining his/her initial block type. The 20 trials in each block consisted of 10 trials with no change and 1 trial with a change in each of the 10 possible positions. The presen- 
Table 3

Proportion of Correct Responses as a Function of Interstimulus Interval and Reflection of Characters in Experiment 3

\begin{tabular}{|c|c|c|c|c|c|c|}
\hline \multirow[b]{3}{*}{ Change } & \multicolumn{6}{|c|}{ Interstimulus Interval } \\
\hline & \multicolumn{2}{|c|}{$34 \mathrm{msec}$} & \multicolumn{2}{|c|}{$67 \mathrm{msec}$} & \multicolumn{2}{|c|}{$217 \mathrm{msec}$} \\
\hline & Normal & Reflected & Normal & Reflected & Normal & Reflected \\
\hline No & .93 & .93 & .73 & .74 & .80 & .77 \\
\hline Yes & .83 & .83 & .62 & .71 & .51 & .58 \\
\hline
\end{tabular}

tation order was individually randomized at the beginning of each block. The general procedure followed that of Experiment 1 .

\section{Results}

Table 3 shows subjects' average accuracy as a function of ISI and whether or not the characters in the display were reflected. A sensitivity analysis of the results was performed. The average $d^{\prime}$ scores are shown in Figure 3. An ANOVA on the $d^{\prime}$ scores indicated that the effect of reflection was nonsignificant $[F(1,17)=.58$, $p>.45]$. The effect of ISI was significant $[F(2,34)=$ $77.6, p<.001]$. The ISI did not significantly interact with reflection $[F(2,34)=2.38, p>.10]$.

\section{Discussion}

The results show that reflection of the characters about a horizontal axis had no significant effects on performance. This conclusion holds for the short ISI (34-msec) condition, in which subjects showed a very high level of performance, perhaps based on detection of motion or other peripheral sensory events. The conclusion also holds for the modest asymptotic performance at 67- and 217msec ISIs. Thus, change detection at these longer ISIs presumably reflects visual short-term memory storage, very likely the same system as that delineated by Phillips (1974). The present results suggest that this storage system may not in any way depend upon, or utilize, the identification of the visual forms as alphanumeric or as other

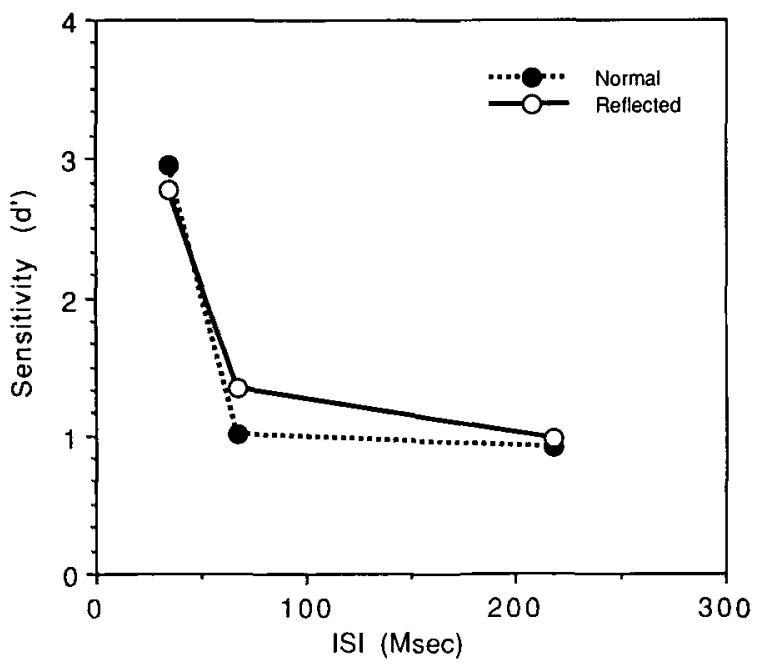

Figure 3. Sensitivity as a function of interstimulus interval (ISI) and reflection of characters in Experiment 3. familiar objects. In the General Discussion, some possible accounts of this will be considered.

It is worth noting that this result mirrors a finding by Hochberg (1968) that involves detecting changes in displays of faces. He found that luminance reversal (as in a photographic negative) did not affect change detection unless the ISI was long. This may have similar implications, although faces do not seem to contain the same sort of discrete object identity by position pairings that alphanumeric displays contain.

\section{EXPERIMENT 4}

Experiment 3 showed that the detection of a change in a display of 10 characters is not much affected by whether the characters are reflected about the horizontal axis. The results appear to rule out any substantial role for abstract letter identity information in the detection of a change from one display to the next. Reflected letters are not entirely unfamiliar, however, and subjects may succeed in noticing from what character a reflected figure is derived, and thus code the display in terms of abstract identities despite the distortion. It is scarcely plausible that such a recoding operation would proceed as rapidly as ordinary character identification. However, if such recoding were operating, and operating more slowly, the previous experiment might have failed to reveal the effect. This might have occurred if, for instance, performance were subject to a ceiling due to the capacity limits of the memory system involved. The presence of a ceiling effect of exactly this sort was noted in the whole-report task studies of Sperling (1960), who pointed out that legibility and spacing had little effect on the whole-report span once displays were presented for intervals long enough to produce asymptotic whole-report performance. Conceivably, an effect like this might have been going on in Experiment 3: subjects might have recoded the items as alphanumeric characters, consuming extra time due to their reflection, but the extra time involved might have been obscured by the fact that performance was simply not exposureduration limited. The present experiment, therefore, compared normal and reflected displays at two different exposure durations (150 and $300 \mathrm{msec}$ ). Since this variation in exposure duration does affect performance, it is not plausible that the rate of identification has no effect upon accuracy.

\section{Method}

Subjects. Twelve undergraduates at the University of California, San Diego, participated as subjects in the 1-h experiment, in partial fulfillment of a course requirement.

Apparatus and Stimuli. The apparatus and stimuli were identical to those of Experiment 3. The main difference was in the stimuli used in the upside-down character blocks. The stimulus set included the digits 4, 7, and 9, in addition to the stimuli of Experiment 3.

Design and Procedure. The experiment was divided into eight blocks, each of which included $\mathbf{4 0}$ trials. There were four block types, reflecting two factors: stimulus duration (150 and $300 \mathrm{msec}$ ), and reflection (normal or reflected). The ISI was $67 \mathrm{msec}$. The eight blocks in the experiment cycled twice through the four block types. 
Table 4

Proportion of Correct Responses as a Function of Stimulus Duration and Reflection of Characters in Experiment 4 Stimulus Duration

\begin{tabular}{cccccc}
\cline { 2 - 3 } & \multicolumn{2}{c}{$150 \mathrm{msec}$} & & \multicolumn{2}{c}{$300 \mathrm{msec}$} \\
\cline { 2 - 3 } \cline { 5 - 6 } Change & Normal & Reflected & & Normal & Reflected \\
\hline No & .80 & .74 & & .80 & .78 \\
Yes & .55 & .58 & & .59 & .63 \\
\hline
\end{tabular}

The subjects were assigned to one of four conditions, determining the duration and familiarity of their initial block. As in the other experiments, the $\mathbf{4 0}$ trials in each block consisted of 20 trials with no change and 2 trials with a change in each of the 10 possible positions. The presentation order was individually randomized at the beginning of each block. The general procedure followed that of Experiment 1.

\section{Results}

The mean performance in each of the four conditions is presented in Table 4 . The results were analyzed in terms of sensitivity $\left(d^{\prime}\right)$ and subjected to an ANOVA. For the 300 -msec exposure duration, the mean $d^{\prime}$ scores were 1.14 and 1.15 for normal and reflected characters, respectively. For the 150-msec exposure duration, the mean $d^{\prime}$ scores were 1.00 and 0.89 for normal and reflected characters, respectively. The ANOVA showed a significant effect of duration $[F(1,11)=7.9, p<.02]$, but no significant effect of reflection $[F(1,11)=.47, p>.50]$. The interaction of duration with reflection was also nonsignificant $[F(1,11)=.45, p>.50]$.

\section{Discussion}

As in the previous experiment, reflection of the characters about a horizontal axis had no significant effect on accuracy of change detection. This result does not depend upon exposure duration, even though the duration variable does have an effect. As pointed out above, this strongly suggests that the lack of an effect of reflection is not produced by a situation in which the reflected characters are being coded in terms of their character identities, albeit more slowly. In short, the results therefore suggest that change detection is mediated by preservation of visual codes that are little affected by familiarity; knowledge of which object identities were presented in which positions in the display does not appear to contribute to performance.

\section{EXPERIMENT 5}

Experiment 4 demonstrated that detection of changes in displays of characters was not impaired by reflection, even when the exposure duration was shortened to $150 \mathrm{msec}$. This extended the result of Experiment 3, and provided some assurance against the possibility that reflection effects were being washed out by asymptotically long exposure to the stimulus. Neither experiment, however, provided any direct assurance that reflection produces a large decrement in identifiability. In fact, doubts previously have been raised about whether this form of distor- tion is an adequate manipulation of identifiability (Krueger, 1975). One might reasonably demand assurance on this point, especially since in a matching task involving single letters, Ambler and Proctor (1976) showed that inversion had no effect unless mixed and inverted letters were mixed within a block.

To directly test the assumption that the present reflection manipulation impairs identification, a new task was used that required subjects to identify all the characters in these displays: subjects determined whether a display contained all characters of the same category, letters or digits (same category), or instead contained a mixture of letters and digits (mixed category). Reflected and normal displays were basically the same as those in the previous experiment.

\section{Method}

Subjects. Twelve undergraduates at the University of California, San Diego, participated as subjects in the 1-h experiment, in partial fulfillment of a course requirement.

Apparatus and Stimuli. The apparatus and stimuli were identical to those of Experiment 4, except as noted. Same-category displays were composed of either 10 letters or 10 digits, selected randomly. This required haphazard repetition of items in the displays, especially in the all-digit displays. Mixed-category displays were created by taking a same-category display and replacing a single item with a character drawn from the opposite category.

Design and Procedure. The experiment was divided into eight blocks, each of which included 40 trials. There were two block types, representing the reflection variable (normal or reflected). The stimulus duration was $300 \mathrm{msec}$. The eight blocks in the experiment cycled four times through the two block types. The subjects were assigned to one of two conditions, determining the duration and familiarity of their initial block. The $\mathbf{4 0}$ trials in each block consisted of 20 trials with same-category displays and 20 trials with mixed-category displays. The presentation order was individually randomized at the beginning of each block.

The subjects were instructed in this experiment to respond as quickly and rapidly as possible, so that both response times and accuracy could be examined.

\section{Results}

Reaction times greater than $2 \mathrm{sec}$ were deleted as anomalous. The mean performance in each of the four conditions is presented in Table 5. Errors were significantly higher $[F(1,11)=103.1, p<.001]$ in the reflected condition $(40.4 \%)$ than in the normal condition (22.9\%). The other error effects were nonsignificant. The reaction times were higher in the reflected condition

Table 5

Reaction Times and Error Rates in Experiment 5

\begin{tabular}{lcc}
\hline & \multicolumn{2}{c}{ Characters } \\
\cline { 2 - 3 } & Normal & Reflected \\
\hline \multicolumn{3}{c}{ Reaction Times (in msec) } \\
Same Category & 1124 & 1178 \\
Mixed Category & 1080 & 1163 \\
& Error Proportions & \\
Same Category & .18 & .40 \\
Mixed Category & .27 & .41 \\
\hline
\end{tabular}


$(1,170 \mathrm{msec})$ than in the normal condition $(1,102 \mathrm{msec})$; this effect was also significant $[F(1,11)=9.6, p<.01]$.

\section{Discussion}

The results confirm the informal observation that reflection of the characters makes it dramatically more difficult for subjects to identify the elements in the display. Considered in conjunction with the results of the previous two experiments, the evidence indicates that change detection is unaffected by processes extracting identities of familiar characters.

\section{GENERAL DISCUSSION}

The overall results provide several empirical generalizations about observers' abilities to detect changes in character arrays. (1) Overall performance with moderate $(67-\mathrm{msec})$ ISIs suggests preservation of no more information than would be revealed in a typical whole-report task. (2) Increasing the duration of the initial display and of the test display produces only a modest improvement in performance. (3) Much better accuracy can, however, be obtained by using very brief ISIs ( $34 \mathrm{msec}$ ); this superior performance is subject to strong effects of intervening masks, apparently without regard to the presence or absence of salient contours in the masking stimulus. (4) Performance is not affected by the reflection of alphanumeric stimuli about the horizontal axis; this conclusion holds equally for conditions in which exposure duration limits performance.

The results seem, therefore, to provide an unambiguously negative answer to the query that motivated the experiments: whether detection of change from one display to the next is powerfully facilitated by "categorical" knowledge of the identities of the objects present at each location in the array. Such facilitation is not evident in the present experiments.

First of all, these four generalizations would appear to be of some interest in the task of simply characterizing the basic functional abilities of human visual information processing. As observers, we have the clear sense of apprehending the identities and locations of large numbers of objects in a scene, and one naturally tends to presume that this sense of apprehension would be accompanied by the ability to detect alterations in these scenes after extremely brief offsets (e.g., $67 \mathrm{msec}$ ). This turns out to be false. At a more theoretical level, the results strongly suggest that subjects perform the present task by using the same kinds of memory representations that are evident in experiments requiring subjects to detect a change in a display of unfamiliar visual stimuli (e.g., Phillips, 1974). Phillips's work has provided an impressive start at characterizing the properties of visual short-term storage, and distinguishing it from sensory memory. Apparently, under the present conditions, visual short-term memory capacity does not depend much upon familiarity or categorization. This observation should obviously not be taken to imply that the memory traces are extremely low level or "pixel-like"; more likely, organized struc- tural descriptions are involved. Indeed, Phillips suggested that visual short-term memory is "schematic," and Hinton (1979) provided evidence for the role of structural descriptions in mental imagery, which may very well reflect the operation of the same system. Therefore, factors affecting the ease with which the structure of a form can be described might determine the difficulty of retaining it in visual short-term memory, even if category labels themselves per se play no role.

At a theoretical level, the results also bear on the issue of automatic identification of familiar items. As described in the introduction, various theorists in the field of attention (e.g., Shiffrin, 1976) have proposed that when adequate sensory information exists, categorization of familiar stimuli proceeds without attentional control or attentional limitations. Furthermore, it is usually assumed that this automatic identification yields a representational structure from which these identities can be retrieved on the basis of various different selection criteria, including location. For instance, if items are presented for a sufficient time to permit the automatic analysis to be completed, then selection by color or location is said to operate without any need to repeat the identification process (Duncan, 1981). It often seems to be assumed that evidence in favor of parallel identification in tasks such as visual search supports this very strong hypothesis. However, it should be emphasized that there are a variety of possible accounts that would postulate parallel identification (e.g., in a search task), while denying the stronger hypotheses of (1) absence of attentional control and (2) creation of a maplike representation from which "late selection" can then operate. One such account is presented and discussed by Pashler and Badgio (1985, 1987).

The present results bear on this issue because they represent one obvious situation in which an automatic process that ties abstract identities to locations might well have revealed itself. That is, if a maplike representation were created, complete with abstract identities and their locations, it might well permit the system to detect changes in the relationship between abstract identities and positions, that is, a change in what object was present in a given position. The present experiments have provided no evidence for any such capability, and thus they are consistent with the view that such maplike representations are not ordinarily created at all (cf. Pashler \& Badgio, 1985). Of course, it would not be correct to describe the present work as a critical test of the maplike representation hypothesis (experiments directed at such a critical test, yielding results that seem to falsify the hypothesis, were reported by Pashler, 1984). For one thing, it is possible that an automatic extraction of identity-location pairs does occur, but that the resulting representation is not of adequate quality or detail to support the judgments required here, forcing the subject to fall back on a lower level visual representation. Another possibility is that while each display is present, subjects might create a temporary representation by tying identities to positions; when a new display appears, however, the earlier representation might 
simply be overwritten, without the system's having any machinery capable of determining whether the old representation and the new one were identical. In either case, the absence of such a capability is plainly a significant and, from many points of view, surprising characteristic of the system.

Another question of interest concerns the relationship between the memory representations underlying performance in this task and those underlying whole-report performance. It was noted above that the level of performance in the two tasks appears to be similar, although there is no way of making as firm a comparison as one would like. Many introductory textbooks suggest that the capacity limit in whole-report performance pertains to the shortterm memory system, which is described as containing "speechlike" (acoustic, auditory, and/or articulatory) codes. In fact, this conclusion is probably incorrect, at least with respect to immediate whole-report performance. Scarborough (1972) looked for evidence of interference between retention of spoken numbers and visual displays of letters and digits. The report of the visual display was written, and the report of the auditory load was spoken. Remarkably, there was no interference between the retention of the two types of material: both could be retained together as effectively as alone. One natural account is that whole report relies on visual coding, presumably of the same sort as that indicated in the present experiments. This sort of hypothesis was initially advanced by Coltheart (1972) and Henderson (1972), although Coltheart (1980) raised various doubts about the hypothesis. The present results certainly increase its plausibility, by arguing for visual encoding of familiar characters, operating with an apparently similar capacity limit to that observed in whole report. Unfortunately, it is difficult to apply the sort of manipulations employed here to the whole-report task. For instance, one would naturally wish to compare whole report for familiar and reflected or otherwise unfamiliar characters; however, this would almost certainly generate severe output interference problems for the subject and scoring problems for the experimenter. For the moment, then, the present results lend indirect support to the hypothesis that whole report is based upon the visual short-term memory representations of Phillips (1974); further research is needed to test this possibility.

\section{REFERENCES}

Ambler, B. A., \& Proctor, R. (1976). The familiarity effect for singleletter pairs. Journal of Experimental Psychology: Human Perception \& Performance, 2, 222-234.

Averbach, E., Coriell, A. S. (1961). Short-term memory in vision. Bell Systems Technical Journal, 40, 309-328.

Avon, S. E., Phillups, W. A. (1980). Visualization and memorization as a function of display time and poststimulus processing time. Journal of Experimental Psychology: Human Learning \& Memory, 6, 407-420.

Coltheart, M. (1972). Visual information processing. In P. C. Dodwell (Ed.), New horizons in psychology. Harmondsworth, England: Penguin Books.
Coltheart, M. (1980). Iconic memory and visible persistence. Perception \& Psychophysics, 27, 183-228.

DunCAN, J. (1980). The locus of interference in the perception of simultaneous stimuli. Psychological Review, 87, 272-300.

Duncan, J. (1981). Directing attention in the visual field. Perception \& Psychophysics, 33, 533-547.

Henderson, L. (1972). Visual and verbal codes: Spatial information survives the icon. Quarterly Journal of Experimental Psychology, 24, 439-447.

Hinton, G. (1979). Some demonstrations of the effects of structural descriptions in mental imagery. Cognitive Science, 3, 231-250.

HochberG, J. (1968). In the mind's eye. In R. N. Haber (Ed.), Contemporary theory and research in visual perception (pp. 309-331). New York: Holt, Rinehart \& Winson.

KRUEGER, L. (1975). Familiarity effects in visual information processing. Psychological Bulletin, 82, 949-974.

KrUeger, L. (1978). A theory of perceptual matching. Psychological Review, 85, 278-304.

KRUEGER, L. (1984). Self-termination in same-different judgments: Multiletter comparison with simultaneous and sequential presentation. Journal of Experimental Psychology: Learning, Memory, \& Cognition, 10, 271-285.

LABERGE, D., \& SAmuels, S. J. (1974). Toward a theory of automatic information processing in reading. Cognition Psychology, 6, 293-323.

LefToN, L. A. (1973). Guessing and the order of approximation effect. Journal of Experimental Psychology, 101, 401-403.

PASHLer, H. (1984). Evidence against late selection: Stimulus quality effects in previewed displays. Journal of Experimental Psychology: Human Perception \& Performance, 10, 429-448.

Pashler, H., \& BADGIO, P. (1985). Visual attention and stimulus identification. Journal of Experimental Psychology: Human Perception \& Performance, 11, 105-121.

Pashler, H., \& Badgio, P. (1987). Attentional issues in the identification of alphanumeric characters. Attention \& performance XII: The psychology of reading. Hillsdale, NJ: Erlbaum.

Philuss, W. A. (1974). On the distinction between sensory storage and short-term visual memory. Perception \& Psychophysics, 16, 283-290.

Phillips, W. A., \& Christie, D. F. M. (1977). Components of visual memory. Quarierly Joumal of Experimental Psychology, 29, 117-133.

Proctor, R. (1981). A unified theory for matching-task phenomena. Psychological Review, 88, 291-326.

Purdy, J., Eimann, D., \& Cross, H. (1980). Persistence of a briefly presented visual stimulus in sensory memory. Bulletin of the Psychonomic Society, 16, 374-376.

Reicher, G., SNyder, C. R. R., \& Richards, J. T. (1976). Familiarity of background characters in visual scanning. Joumal of Experimental Psychology: Human Perception \& Performance, 2, 522-530.

SCARborough, D. L. (1972). Memory for brief visual displays of symbols. Cognitive Psychology, 3, 408-429.

SHIFFRIN, R. M. (1976). Capacity limitations in information processing, attention, and memory. In W. K. Estes (Ed.), Handbook of learming and cognitive processes (Vol. 4, pp. 177-236). Hillsdale, NJ: Erlbaum.

SPERLING, G. (1960). The information available in brief visual presentations. Psychological Monographs, 74(11, Whole No. 498).

\section{NOTE}

1. An informal test of phosphor persistence, which involved viewing static displays of this type through an electronic shutter, indicated that visible persistence was on the order of $5 \mathrm{msec}$ (based on the size of the subset of a full-screen display that was visible through a very brief shutter exposure). This estimate is rough, and nothing in the conclusions would seem to depend upon its precision; in any case, details on the assessment method employed are available from the author.

(Manuscript received April 8, 1987; revision accepted for publication April 4, 1988.) 